\title{
Histopathological and Immunohistochemical Evaluation of Primary Muscular Peripheral T Cell Lymphoma in a Dog
}

\author{
Carlos Eduardo Fonseca-Alves', Marina Gabriela Possa², Fabrício Bernardi², \\ Helvécio Leal Santos-Junior ${ }^{3}$, Rômulo Santos Adjuto Eloi ${ }^{4}$ \& Fabiana Elias²
}

\begin{abstract}
Background: Canine lymphoma is the most common hematopoietic neoplasm in dogs and reveals divergent biological behaviors correlated to histopathological subtype, the immunophenotypic ( $\mathrm{T}$ or B) and tumor stage. The multi-centric form is the most common presentation for canine lymphoma, followed by gastrointestinal and cutaneous forms. Miscellaneous forms of canine lymphoma (nasal, osseous, central nervous system and muscle) represent less than $1 \%$ of all cases. This report describes the clinical, macroscopic, histopathological and immunohistochemical findings detected in a dog with a primary muscular lymphoma.

Case: The subject was referred to the Emergency and Critical Care Service at the Veterinary Hospital with a history of claudication in the left pelvic limb, severe dehydration, hypovolemia, vomiting and diarrhea caused by gastroenteritis associated with the use of phenylbutazone. After death, the post-mortem examination revealed ulcerative gastritis in the gastrointestinal tract (GIT). Histopathological examination of the GIT specimens, mesenteric lymph nodes, and the left popliteal lymph node revealed no neoplastic alterations. Histological examination of semitendinosus muscle revealed proliferation of cells with round or oval nucleus, an evident pleomorphic nucleolus and scanty, eosinophilic cytoplasm. There were five to six mitosis per each 400x field. These cells infiltrated through the muscle fibers. The muscle fibers displayed marked eosinophilic sarcoplasm, loss of striations and fragmentation (degeneration). Immunohistochemical staining revealed negative reaction for CD79a and positive for CD45 and CD3.

Discussion: The primary muscle lymphoma it is very rare disease and patients commonly have clinical signs related with muscle location. Our description of muscular primary lymphoma affecting the semitendinosus muscle emphasize that it must be included as a differential diagnosis for dogs with unilateral lameness, inflammatory processes, and other malignancies. In this case, the patient showed an ulcerative gastroenteritis associated with the inappropriate use of phenylbutazone. The patient death was associated with a septicemia due to several ulcers in the gastrointestinal tract. We excluded any regional lymph node involvement and secondary muscular infiltration with post-mortem and histopathological examination. The gross evaluation of the left hind limb demonstrated only muscular involvement (semitendinosus muscle) without infiltration in the adjacent structures, and the histopathology revealed no alteration in the regional lymph node. The immunohistochemical evaluation showed negative staining to CD79a, a high number of positive cells to Ki67 and positive staining to CD45 and CD3. In normal lymph nodes, it was possible to note CD79 diffuse expression in germinal centers in lymphoid follicles and few positive B-lymphocytes in medullary region. Diffuse CD3 expression was found in cortex region by normal Tlymphocytes. There was no histological alterations in sublumbar and popliteal lymph nodes. This immunohistochemical and histological patterns revealed a Peripheral T Cell lymphoma with a high proliferative index. The previous report of primary muscular lymphoma showed a $\mathrm{T}$ cell lymphoma with a high proliferative index similar to our findings. Based on macroscopic, histopathological, and immunohistochemical findings it was concluded that the patient had a primary muscular Peripheral T Cell lymphoma.
\end{abstract}

Keywords: canine, histopathology, CD3, CD79a, skeletal muscle. 


\section{INTRODUCTION}

Canine lymphoma is the most common hematopoietic neoplasm in dogs and reveals divergent biological behaviors correlated to histopathological subtype, the immunophenotypic (T or B) and tumor stage [7]. The multi-centric form is the most common presentation for canine lymphoma, followed by gastrointestinal and cutaneous forms [6]. Miscellaneous forms of canine lymphoma (nasal, osseous, central nervous system and muscle) represent less than $1 \%$ of all cases [6]. Canine lymphoma can affect any breed or size; however, there is an overrepresentation among the middle-sized to larger dog breeds [12]. These frequent reports could be related to a genetic susceptibility $[6,12]$ as the Bullmastiff dogs were previously reported with a familial predisposition to lymphoma development [8].

In humans, primary muscular lymphoma or nodal lymphoma infiltrating adjacent muscles is extremely rare, with only isolated case reports in the literature [1]. Human muscle lymphoma occurs with the most frequency in the gluteal and pelvic muscles, spreading from adjacent involved lymph nodes $[1,3,4]$. Previously, eight cases of primary muscle lymphoma out of 2,824 lymphomas were reported in the literature in AIDS-associated non-Hodgkin lymphoma patients [1]. The diagnosis is based on computed tomography (CT) and magnetic resonance imaging (MRI) findings associated with biopsy of the affected muscle and regional lymph node [3]. Due to limited number of published cases, there is no information about the worst prognostic or outcome in patients with primary muscle lymphoma compared to Non-Hodgkin's lymphoma.

Muscular lymphoma is usually associated with neoplastic infiltration from adjacent lymph nodes [4] and primary muscular lymphoma is a rare presentation of this neoplasm, disease with only three previous reports in the Veterinary literature $[5,9,10]$. Therefore, it is necessary to differentiate primary muscle lymphomas from multicentric lymphoma with muscular involvement [9]. The aim of this study was to describe a case of primary muscle lymphoma in a Bullmastiff dog.

\section{CASE}

A 8-year-old entire male Bullmastif dog was referred to the Veterinary Hospital at the School of Veterinary Medicine - UPIS University with a 7-days history of lameness of the left pelvic limb. The owner injected phenylbutazone (Fenilbutazona $\left.\mathrm{OF}^{\circledR}\right)^{1} 10 \mathrm{mg} /$ $\mathrm{kg}$ BID intramuscularly, not based on veterinary advice or guidance for three days in the right pelvic limb. Two days after the medication was administrated, the patient had diarrhea, vomiting, and anorexia and four days after the onset of these clinical signs, the owner went to the Veterinary Hospital. Physical examination revealed a temperature of $39.8^{\circ} \mathrm{C}$, pale mucous membranes, abdominal pain and melena. The patient suffered and from cardiac arrest during clinical care. Resuscitation procedures were performed but the patient died due to sepsis and the post-mortem examination was performed. At necropsy, circular depressions of 0.3 to $0.5 \mathrm{~cm}$ in diameter were observed in the mucosa of the fundus of the stomach. The small intestine revealed diffuse mucosal hyperemia. In the left hind limb, white homogenous masses infiltrating the muscle fibers were observed (Figure 1). There was no involvement of regional lymph nodes or other muscular structures. Specimens from stomach and intestine revealed ulcerative gastroenteritis. Mesenteric and popliteal lymph nodes showed no histopathological alterations. The gross findings suggested sepsis was a cause of death. Tissue specimens from the stomach, intestinal segments, left and right kidneys, spleen, mesenteric, inguinal and prescapular lymph nodes, semitendinosus muscle of the left hind limb, and the left popliteal lymph node were collected and fixed in a $10 \%$ buffered formalin, routinely processed and embedded in paraffin wax. Sections were stained with hematoxylin and eosin (HE). Histological examination of semitendinosus muscle revealed proliferation of cells with round or oval nucleus, an evident pleomorphic nucleolus and scanty, eosinophilic cytoplasm (Figure 2). There were 5 to 6 mitosis per each 400x field. These cells infiltrated through the muscle fibers. The muscle fibers displayed marked eosinophilic sarcoplasm, loss of striations and fragmentation (degeneration).

Histopathological examination suggested a primary muscular lymphoma. To confirm the diagnosis, immunohistochemistry for CD3, CD79, CD45, and Ki67 was performed in the tumor specimens and reginal lymph nodes (sublumbar and popliteus) according to Valli et al. [11]. The classification of the tumor subtype was performed according to the World Health Organization (WHO) criteria [11]. The Ki67 staining $^{2}$ was performed to evaluate the proliferative index. Briefly, the immunohistochemical staining was 


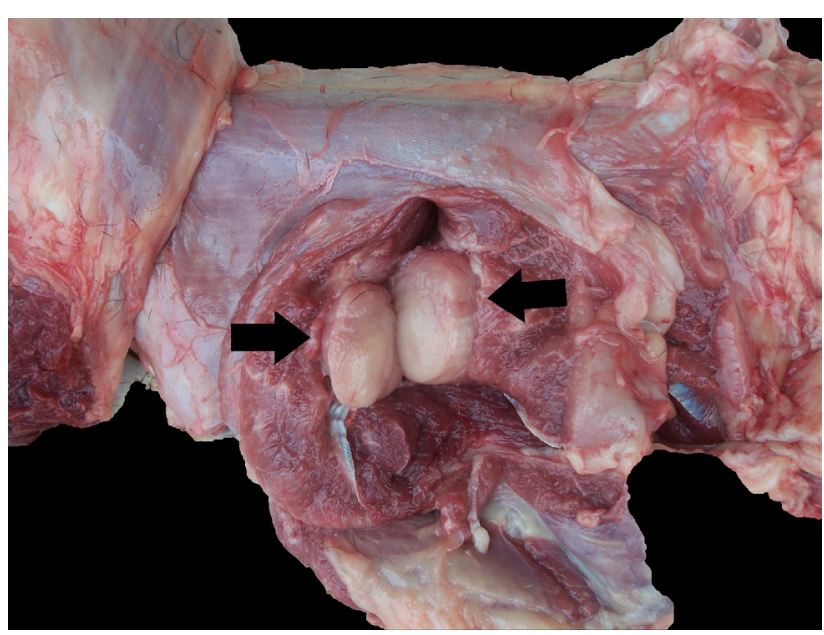

Figure 1. Canine bullmastiff showing a primary muscularT cell lymphoma. Left pelvic limb showing the semitendinosus muscle with white-homogenous masses infiltrating the muscle fibers (black arrows). No infiltration into adjacent muscles.

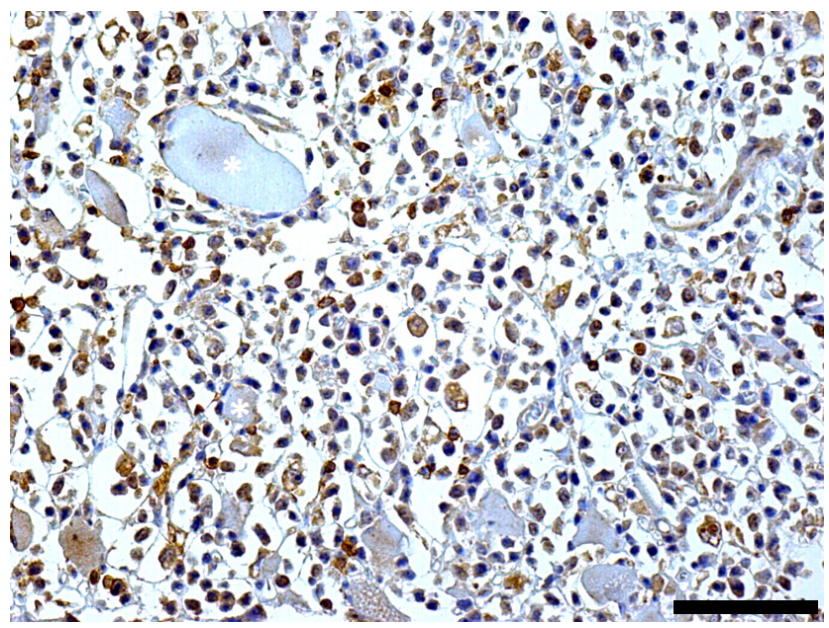

Figure 3. Immunohistochemical staining of $\mathrm{CD} 3$ in semitendinosus muscle showing positive membranous staining in around $80 \%$ of neoplastic cells. [Scale bar $=50 \mu \mathrm{m}]$.

performed using a peroxidase method and 3,3' diaminobenzidine tetrachloride $(\mathrm{DAB})^{3}$. The slides were dewaxed in xylol and rehydrated in graded ethanol. For antigen retrieval, the slides were incubated in a citrate buffer ( $\mathrm{pH}$ 6.0) in a pressure cooker (Pascal $\left.{ }^{\circledR}\right)^{4}$. CD3 was detected with a polyclonal antibody ${ }^{4}$ at a 1 in 100 dilution, applied for $45 \mathrm{~min}$. The antibodies against CD79a and Ki67 were a monoclonal mouse antibody ${ }^{4}$, used at a 1 in 50 dilution for $45 \mathrm{~min}$. CD45 detection was performed with a monoclonal mouse antibody (Clone: CA12.10C12. Isotype: IgG1) ${ }^{2}$, which was used at a 1 in 50 dilution for $45 \mathrm{~min}$. The immunolabeling was performed by the Histofine method $(414154 \mathrm{~F})^{5}$. The sections were examined under light microscope at ×400 magnification. Omission of the primary antibody and replacement with the diluting solution alone was used as a negative control. The positive control con-

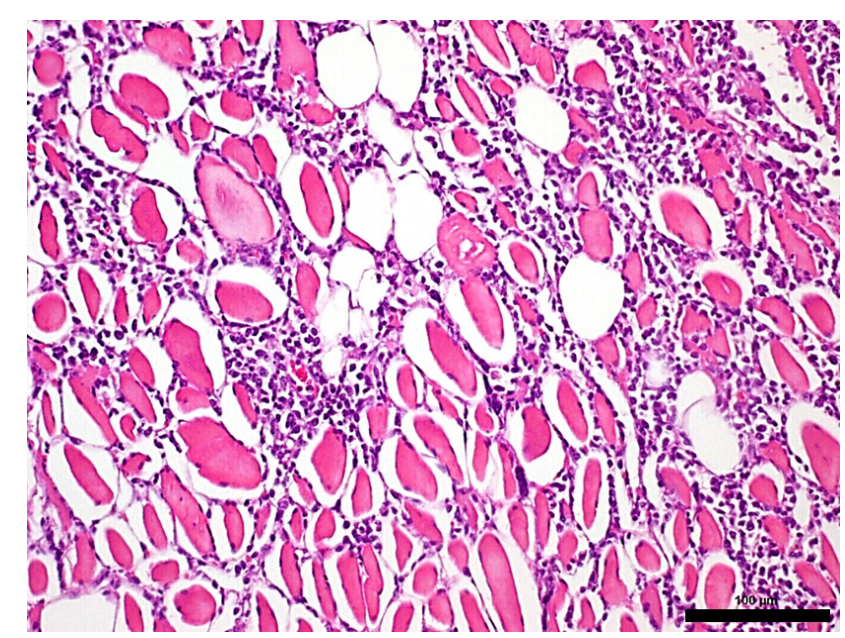

Figure 2. Semitendinosus muscle showing proliferation of neoplastic mononuclear cells with round or oval nucleus, infiltrating the muscle fibers. [Scale bar $=50 \mu \mathrm{m}]$.

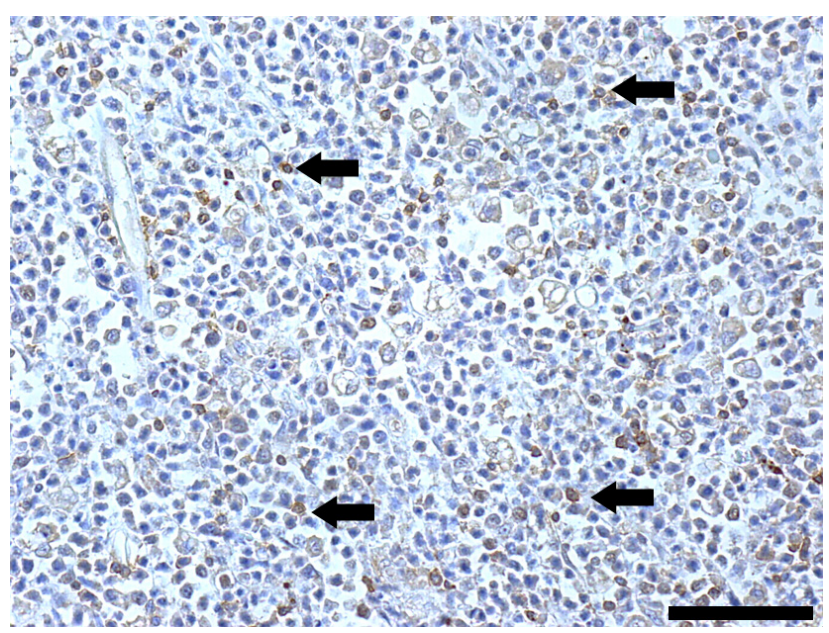

Figure 4. Immunohistochemical staining of CD79a in semitendinosus muscle showing few infiltrating B-cell lymphocyte (black arrows) and negative neoplastic cells for CD79a. [Scale bar $=50 \mu \mathrm{m}$ ].

sisted of a normal canine lymph node for all antibodies. A negative control was performed for all antibodies by omitting the primary antibody and substituting with Tris-buffered saline. Neoplastic cells were positive for CD45 and CD3 (Figure 3) and negative for CD79a, even if scattered infiltrating $B$ cells were detected (Figure 4). We determine the percentage of positive cells for Ki67 staining in a five high power field and we found that more than $90 \%$ of the neoplastic cells showed positive staining.

\section{DISCUSSION}

The primary muscle lymphoma it is very rare disease and patients commonly have clinical signs related with muscle location [1]. Our description of muscular primary lymphoma affecting the semitendinosus muscle emphasize that it must be included as 
a differential diagnosis for dogs with unilateral lameness, inflammatory processes, and other malignancies [5,9]. In this case, the patient showed an ulcerative gastroenteritis associated with the inappropriate use of phenylbutazone. The patient death was associated with a septicemia due to several ulcers in the gastrointestinal tract. We excluded any regional lymph node involvement and secondary muscular infiltration with post-mortem and histopathological examination.

The gross evaluation of the left hind limb demonstrated only muscular involvement (semitendinosus muscle) without infiltration in the adjacent structures, and the histopathology revealed no alteration in the regional lymph node. The immunohistochemical evaluation showed negative staining to CD79a, a high number of positive cells to Ki67 and positive staining to CD45 and CD3. In normal lymph nodes, it was possible to note CD79 diffuse expression in germinal centers in lymphoid follicles and few positive B-lymphocytes in medullary region. Diffuse CD3 expression was found in cortex region by normal T-lymphocytes. There was no histological alterations in sublumbar and popliteal lymph nodes. This immunohistochemical and histological patterns revealed a Peripheral T Cell lymphoma with a high proliferative index. The previous report of primary muscular lymphoma showed a $\mathrm{T}$ cell lymphoma with a high proliferative index similar to our findings $[5,9,10]$.

Ki67 protein it is a nuclear antigen expressed in cycling cells, widely used to establish tumor proliferation [2]. In this case, we identified a high Ki67 index (more than $90 \%$ of Ki67 positive cells), suggesting a tumor with high growth fraction.

The macroscopic, histopathological, and immunohistochemical findings identified a primary muscular Peripheral T Cell lymphoma associated with a high Ki67 expression in a Brazilian Bullmastiff dog. We believe that this case description represents the first report of a primary Peripheral T Cell lymphoma affecting the semitendinosus muscle in a dog.

\author{
MANUFACTURERS \\ ${ }^{1}$ Ouro Fino Saúde Animal, Cravinhos, SP, Brazil. \\ ${ }^{2}$ Abcam. San Francisco, CA, USA. \\ ${ }^{3}$ Sigma-Aldrich Ltda. São Paulo, SP, Brazil. \\ ${ }^{4}$ DakoCytomation California Inc. Carpinteria, CA, USA. \\ ${ }^{5}$ Nichirei Biosciences Inc. Tokyo, Japan.
}

Declaration of interest. The authors report no conflicts of interest. The authors alone are responsible for the content and writing of the paper.

\section{REFERENCES}

1 Beral V., Peterman T., Berkelman R. \& Jaffe H. 1991. AIDS-associated non-Hodgkin lymphoma. Lancet. 337: 806-809. 2 Broyde A., Boycov O., Strenov Y., Okon E., Shpilberg O. \& Bairey O. 2009. Role and prognostic significance of the Ki-67 index in non-Hodgkin's lymphoma. American Journal of Hematology. 84(6): 338-343.

3 Chong J., Som P.M., Silvers A.R. \& Dalton J.F. 1998. Extranodal non-Hodgkin lymphoma involving the muscles of mastication. American journal of neuroradiology. 19(10): 1849-1851.

4 Dai Y., Sowjanya M., You J. \& Xu K. 2015. Non-Hodgkin's Lymphoma of Multiple Skeletal Muscles Involvement Seen on FDG PET/CT Scans. Medicine. 94(18): e833.

5 Harkin K.R., Kennedey G.A., Moore W.E. \& Schoning P. 2000. Skeletal muscle lymphoma in a bullmastiff. Journal of the American Animal Hospital Association. 36(1): 63-66.

6 Helfand S.C. \& Vail D.M. 1993. Hematopoietic system. In: Slatter D. (Ed). Textbook of small animal surgery. 2nd edn. Philadelphia: WB Saunders, pp.2111-2136.

7 Marconato L., Polton G.A., Sabattini S., Dacasto M., Garden O.A., Grant I., Hendrickx T., Henriques J., Lubas G. Morello E. Stefanello D. \& Comazzi S. 2017. Conformity and controversies in the diagnosis, staging and follow-up evaluation of canine nodal lymphoma: a systematic review of the last 15 years of published literature. Veterinary and Comparative Oncology. 15(3): 1029-1040.

8 Onions D.E. 1984. A prospective survey of familial canine lymphosarcoma. Journal of National Cancer Institute. 72(4): 909-912.

9 Takeuchi Y., Fujino Y., Goto-Koshino Y., Ohno K., Uchida K., Nakayama H. \& Tsujimoto H. 2010. Long term survival of primary skeletal muscle lymphoma in a miniature dachshund. The Journal of Veterinary Medical Science. 72(5): 673-677.

10 Thuilliez C., WaTrelot-Viriex D., Chanut F., Fournel-Fleury C., Ponce F. \& Marchal T. 2008. Presumed primary muscular lymphoma in a dog. Journal of Veterinary Diagnostic Investigation. 20(6): 824-826. 
C.E. Fonseca-Alves, M.G. Possa, F. Bernardi, et al. 2017. Histopathological and Immunohistochemical Evaluation ofPrimary Muscular Peripheral T Cell Lymphoma in a Dog. Acta Scientiae Veterinariae. 45(Suppl 1): 242.

11 Valli V.E., San Myint M., Barthel A., Bienzle D., Caswell J., Colbatzky F., Durham A., Ehrhart E.J., Johnson Y., Jones C., Kiupel M., Labelle P., Lester S., Miller M., Moore P., Moroff S., Roccabianca P., Ramos-Vara J., Ross A., Scase T., Tvedten H. \& Vernau W. 2011. Classification of canine malignant lymphomas according to the World Health Organization criteria. Veterinary Pathology. 48(1): 198-211.

12Zandvliet M. 2016. Canine lymphoma: a review. Veterinary Quarterly. 36(2): 76-104. 